\title{
Smart-anesthesia Application with Artificial Intelligence for a Preoperative Evaluation of Patients
}

\author{
Tural Alekberli ${ }^{1}$, Fidan R Alekberli ${ }^{2}$, Chloé Mimouni ${ }^{3}$, Abu A Muhammed ${ }^{4}$
}

\begin{abstract}
Anesthesiology is a dynamic specialty of medicine that fosters continuous improvements in anesthetic care for patients undergoing surgical procedures. The preoperative (pre-op) evaluation of a patient is vital for reasonable and acceptable patient care. Inadequate pre-op patient preparation causes medical, organizational, economic, and emotional problems to patients and professionals. There are no standards for the level of knowledge and skills of an anesthesiologist worldwide; the difference is more dramatic, especially in a country without proper residency education. It can be life-threatening, especially during urgent cases where resident anesthesiologists evaluate patients frequently at the bedside before the procedure. Smart-anesthesia project is an innovative online preoperative anesthesia evaluation application (App), uses artificial intelligence (Al), gives perioperative anesthesia recommendations. The app is available for smartphone/tablet/laptop and compatible with android/IOS/windows, which is useful for elective/urgent procedures. A printable assessment document with an anesthesia recommendation list is available. Compatible with ICD-11 codes to avoid terminology and grammar-related issues. Minimal handwriting for maximal automation. Rapid screening and assessment of patients with a user-friendly interface. Safe practice of anesthesiology with recommendations by analyzing data with updated American Society of Anesthesiologists (ASA) guidelines. An automatic ASA scoring will be available. A universal preoperative assessment tool that connects anesthesiologists around the world with a common language for safer anesthesia practice.

Keywords: Anesthesia assessment, Artificial intelligence, Innovation in anesthesia, Preoperative evaluation.

Research and Innovation in Anesthesia (2020): 10.5005/jp-journals-10049-0078
\end{abstract}

\section{INTRODUCTION}

Anesthesiology is a dynamic specialty of medicine that fosters continuous improvements in anesthetic care for patients undergoing surgical procedures. The preoperative (pre-op) evaluation of a patient is vital for reasonable and acceptable patient care. Inadequate pre-op patient preparation causes medical, organizational, economic, and emotional problems to patients and professionals. ${ }^{1,2}$

The primary goal of pre-anesthetic assessment is to obtain the information required to plan anesthetic management. An anesthesiologist should document all aspects of the patient's medical and surgical history, findings on physical examination, and laboratory investigations relevant to anesthetic management. The patient's history should include past and current medical problems, current and recent drug therapy, unusual reactions or responses to drugs, and any problems or complications associated with previous anesthetics. A family history of adverse reactions related to anesthesia should also be obtained. Information about the anesthetic that the patient considers relevant should also be documented. An American Society of Anesthesiologists (ASA) physical status classification should be recorded for each patient. ${ }^{1}$

\section{Current Problems}

There are no standards for the level of knowledge and skills of an anesthesiologist worldwide; the difference is more dramatic, especially in a country without proper residency education. In those countries, the senior anesthesiologists also may fail in adequately evaluating a patient preoperatively. In most of the anesthesia residency education programs, even in developed countries, the junior (1st-2nd year) residents evaluate patients pre-op alone or with the supervision of attending, which may lead to improper evaluation frequently. It can be life-threatening,
1,3,4 Department of Anesthesiology, Critical Care and Pain Medicine, Hadassah Medical Center, The Hebrew University, Jerusalem, Israel ${ }^{2}$ Department of Otorhinolaryngology, Head and Neck Surgery, Hadassah Medical Center, The Hebrew University, Jerusalem, Israel

Corresponding Author:Tural Alekberli, Department of Anesthesiology, Critical Care and Pain Medicine, Hadassah Medical Center, The Hebrew University, Jerusalem, Israel, Phone: +972 504048435, e-mail: turalalek@gmail.com

How to cite this article: Alekberli T, Alekberli FR, Mimouni C, et al. Smartanesthesia Application with Artificial Intelligence for a Preoperative Evaluation of Patients. Res Inno in Anesth 2020;5(1):21-22.

Source of support: Nil

Conflict of interest:The main author Tural Alekberli is the founder and owner of the Smart-anesthesia Project.

especially during urgent cases where resident anesthesiologists evaluate patients frequently at the bedside before the procedure. Most of the hospitals are having their software to collect data during preoperative evaluation in the clinic. However, they are still using paper records or information leaflets during urgent cases.

\section{Smart-Anesthesia Project}

Smart-anesthesia is an innovative online preoperative anesthesia evaluation application. The first online app that uses artificial intelligence (Al) gives peri-op anesthesia recommendations and contains many futuristic specifications to assist anesthesiologists.

The app is compatible with ICD-11 codes to avoid terminology and grammar-related issues. The app offers the advantage of minimal handwriting for maximal automation and technological compatibility. It is suitable for android, IOS, and windows, whatever the informatics support: smartphone, computer, tablet, and laptop. 
The use of this app and its online platform process of assessment allows a time-saving and efficient pre-op anesthesia evaluation that helps the anesthesiologist team to focus on clinical decisions. The app allows rapid screening and assessment of patients with a user-friendly interface, which allows for better planning of patient operations by improving and reducing the referral to treatment time pathway. Smart-anesthesia guarantees a safe practice of anesthesiology: it reminds physicians of pre-op anesthetic issues and gives them recommendations by analyzing entered data with updated ASA and European Society of Anesthesiology and Intensive Care (ESAIC) guidelines. Smart-anesthesia enhances patient safety for emergent surgeries thanks to a quick and detailed bedside evaluation, carefully analyzed by the smart system, which delivered an automatic ASA scoring with the perioperative recommendations. Patient's data are now easy to collect and to study for healthcare facilities and anesthesiologists. Indeed, the pre-op anesthesia form (PDF File) and the peri-op recommendations (PDF File) are ready to print and stored on a cloud (Fig. 1). Operating room morbidity and complications rate worldwide could be decreased only by focusing on pre-op assessment and assisting anesthesiologists in the field day and night. The primary purpose of this app is to create a global and universal pre-op assessment tool that connects anesthesiologists around the world with a single common language for safer anesthesia practice.

\section{References}

1. Dubson G, Chow L, Flexman A, et al. Guidelines to the practice of anesthesia - revised edition. Can J Anesth 2019;66(1):75-108. DOI: 10.1007/s12630-018-1248-2.

2. Herrera-Usagre M, Santana V, Burgos-Pol R, et al. Effect of a mobile app on preoperative patient preparation for major ambulatory

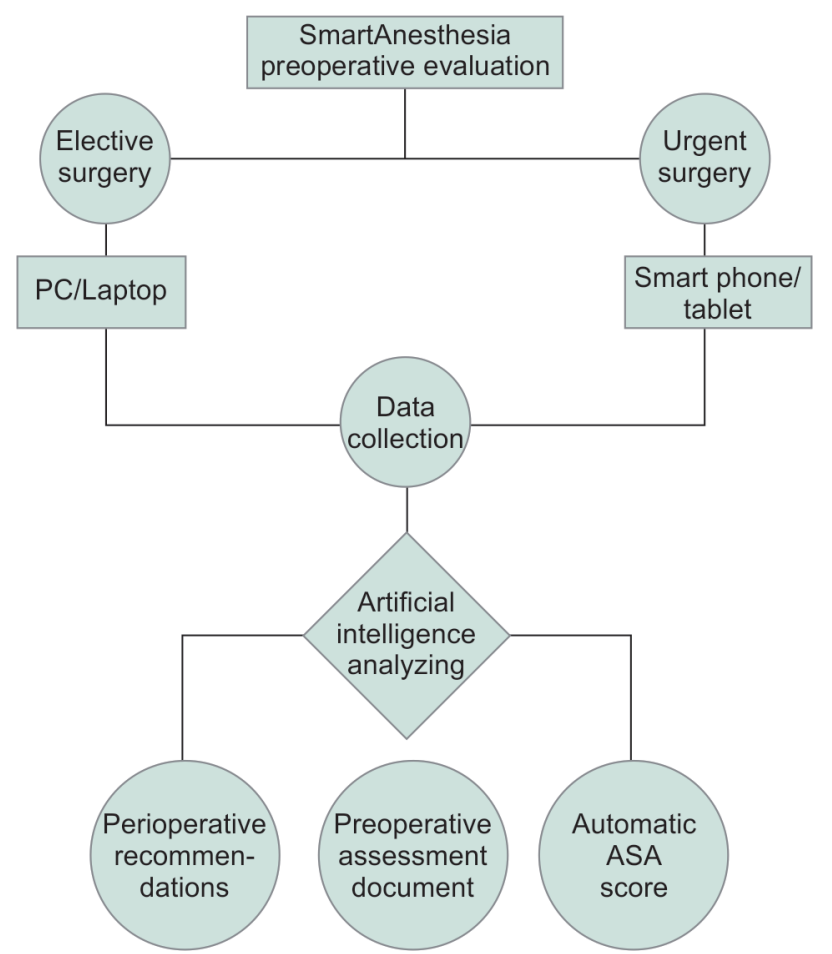

Fig. 1: A brief explanation of the preoperative anesthesia assessment process by using a smart-anesthesia application

surgery: protocol for a randomized controlled trial. JMIR Res Protoc 2019;8(1):e10938. DOI: 10.2196/10938. 